\title{
Companion varieties for root systems and Fermat arrangements
}

\author{
Roberta Di Gennaro, Giovanna Ilardi, Rosa Maria Miró-Roig \\ Tomasz Szemberg, Justyna Szpond
}

February 9, 2022

\begin{abstract}
Unexpected hypersurfaces are a brand name for some special linear systems. They were introduced around 2017 and are a field of intensive study since then. They attracted a lot of attention because of their close connections to various other areas of mathematics including vector bundles, arrangements of hyperplanes, geometry of projective varieties. Our research is motivated by what is now known as the BMSS duality, which is a new way of deriving projective varieties out of already constructed ones. The last author coined the concept of companion surfaces in the setting of unexpected curves admitted by the $B_{3}$ root system. Here we extend this construction in various directions. We revisit the configurations of points associated to either root systems or to Fermat arrangements and we study the geometry of the associated varieties. In the case of configurations of points associated to root systems, the geometry of their companions is also described.
\end{abstract}

\section{Introduction}

In the present note we study companion varieties of unexpected hypersurfaces associated to some symmetric configurations of points in projective spaces. Companion varieties were introduced by the last author in [26, Section 4.4] in the course of her study of Togliatti-type surfaces.

It is a classical problem in algebraic geometry to determine the dimension of linear systems. A lot of attention was given to linear systems with imposed base loci, i.e., of the form

$$
H^{0}(X ; L \otimes \mathcal{I}(Z))
$$

where $L$ is a positive (e.g. ample or very ample) line bundle on a smooth variety $X$ and $Z$ is a subscheme of $X$. Even in the very simple situation when $X$ is the projective plane, $L$ is the line bundle $\mathcal{O}_{\mathbb{P}^{2}}(d)$ for $d>0$ and $Z$ is a zero-dimensional subscheme of $\mathbb{P}^{2}$ the problem of computing the dimension of the vector space in (1) is far from being completely understood. Indeed, the two open conjectures: one due to Nagata (1959) [20] and the other the SHGH-Conjecture package due to Segre (1969), Harbourne (1986), Gimigliano (1987) and Hirschowitz (1989) (see [8] for introduction and recent progress) provide sufficient evidence for this claim. In any case, it is well-understood and clear that a single point, or a fat point scheme concentrated in a single general point impose independent conditions on homogeneous polynomials of any fixed degree in a projective space of arbitrary dimension. With this in mind, it came as a surprise when Cook II, Harbourne, Migliore and Nagel

\footnotetext{
Key words and phrases. unexpected hypersurfaces, arithmetically Cohen-Macaulay varieties, root systems, Fermat-type arrangements, Veronese varieties.

2010 Mathematics subject classification. 13A15, 13C70, 14C20, 14E05, 14J70, 14N20
} 
[3], inspired by earlier results due to Di Gennaro, Ilardi and Vallès [5], announced that a single general fat point might impose fewer conditions than expected on the linear system of homogeneous polynomials with assigned base loci. The elements arising this way are called unexpected hypersurfaces, see Section 2 for precise definitions.

The unexpected hypersurfaces are interesting not only because they violate the naive conditions count when determining the dimension of a linear system. In a private communication to the last author Igor Dolgachev suggested that, under additional positivity conditions, there should be a relation between the existence of unexpected hypersurfaces and varieties with defective osculating spaces. This has been indeed established in [27, Section 4.2] for a configuration of points determined by the $B_{3}$ root system. The linear system of quadrics vanishing at configuration points determines, after passing to the blow up of $\mathbb{P}^{2}$ in these points, a morphism to $\mathbb{P}^{5}$ whose image is a surface $S$ such that at every point $P$ of $S$, there is a hyperplane in $\mathbb{P}^{5}$ tangent to $P$ to order 2 (in other words: cutting out on $S$ a curve which passes through $P$ with multiplicity at least 3 ). It has been observed additionally, that there is another surface $S^{\prime}$, which we call a companion surface of $S$, which exhibits also interesting geometrical properties. This example motivates our present work.

The general yoga, in the basic version taken from [27], is as follows. Assume that there is a set of points $Z$ in $\mathbb{P}^{N}$ which admits a unique unexpected hypersurface $H_{Z, P}$ of degree $d$ and multiplicity $m$ at a general point $P=\left(a_{0}: \cdots: a_{N}\right) \in \mathbb{P}^{N}$. Let

$$
F_{Z}\left(\left(a_{0}: \cdots: a_{N}\right),\left(x_{0}: \cdots: x_{N}\right)\right)=0
$$

be a homogeneous polynomial equation of $H_{Z, P}$. Let $g_{0}, \ldots, g_{T}$ be a basis of the vector space $[I(Z)]_{d}$ of homogeneous polynomials of degree $d$ vanishing at all points of $Z$. Under some mild hypotheses the unexpected hypersurface $H_{Z, P}$ comes from a bi-homogeneous polynomial $F_{Z}\left(\left(a_{0}: \cdots: a_{N}\right),\left(x_{0}: \cdots: x_{N}\right)\right)$ of bidegree $(m, d)$, see [17, Section 4] and [7, Proposition 1]. Indeed, $F_{Z}$ can be written in a unique way as a combination

$$
F_{Z}=h_{0}\left(a_{0}: \cdots: a_{N}\right) g_{0}\left(x_{0}: \cdots: x_{N}\right)+\cdots+h_{T}\left(a_{0}: \cdots: a_{N}\right) g_{T}\left(x_{0}: \cdots: x_{N}\right)
$$

where $g_{0}\left(x_{0}: \cdots: x_{N}\right), \ldots, g_{T}\left(x_{0}: \cdots: x_{N}\right)$ are homogeneous polynomials of degree $d$ forming the basis mentioned above and $h_{0}\left(a_{0}: \cdots: a_{N}\right), \ldots, h_{T}\left(a_{0}: \cdots: a_{N}\right)$ are homogeneous polynomials of degree $m$. Therefore, there are two rational maps naturally associated to equation (2)

$$
\varphi: \mathbb{P}^{N} \ni\left(x_{0}: \cdots: x_{N}\right) \rightarrow\left(g_{0}\left(x_{0}: \cdots: x_{N}\right): \cdots: g_{T}\left(x_{0}: \cdots: x_{N}\right)\right) \in \mathbb{P}^{T}
$$

and

$$
\psi: \mathbb{P}^{N} \ni\left(a_{0}: \cdots: a_{N}\right) \rightarrow\left(h_{0}\left(a_{0}: \cdots: a_{N}\right): \cdots: h_{T}\left(a_{0}: \cdots: a_{N}\right)\right) \in \mathbb{P}^{T} .
$$

The image of $\psi$ is the companion variety of the image of $\varphi$. The purpose of this note is to study properties of companion varieties and relations between them. Turning to details in Sections 4 and 5 we work in a more general situation where $g_{0}, \ldots, g_{T}$ form a basis of a vector subspace in $[I(Z)]_{d}$ large enough to write down $F_{Z}$ in the form in (2). It might also happen that the coefficients $h_{0}, \ldots, h_{T}$ are linearly dependent. In this case we work rather with their linearly independent subset in order to avoid dealing with degenerate subvarieties. Thus it is possible (and it actually happens) that $\varphi$ and $\psi$ are mappings to projective spaces of different dimensions.

We focus here on three natural generalizations of the aforementioned $B_{3}$ configuration. In what follows $H$ is always the pull-back of the hyperplane bundle under the appropriate blow up. 
First, results from [17] suggest that all $B_{n}$ configurations give rise to unexpected hypersurfaces in $\mathbb{P}^{n-1}$. In case of $n=4$ there is also a unique unexpected hypersurface and we determine its explicit equation in (4) and in slightly different generators in (6). It turns out that the unexpected surface for $B_{4}$ is also unexpected for $F_{4}$, a non-crystallographic root system. The other difference when compared to the $B_{3}$ root system is that now the degree of the unexpected surface equals its multiplicity in the general point, i.e., the unexpected surfaces are cones.

Theorem $B_{4} / F_{4}$. Let $Y$ be the blow up of $\mathbb{P}^{3}$ at points in the $F_{4}$ root system with exceptional divisors $E_{1}, \ldots, E_{24}$. Then the linear system $4 H-\sum E_{i}$ embeds $Y$ into $\mathbb{P}^{11}$ as a smooth threefold $X$ of degree 40. The companion threefold $X^{\prime}$ is in this case isomorphic to $X$.

This is proved in Proposition 4.3 and Remark 4.4.

Secondly, we study the $H_{3}$ root system. Similarly as $B_{3}$ for $m=3$, it admits an unexpected curve of degree $m+1$ with multiplicity $m$ at the general point, this time with $m=5$.

Theorem $H_{3}$. Let $Y$ be the blow up of $P^{2}$ at points in the $H_{3}$ root system with exceptional divisors $E_{1}, \ldots, E_{15}$. Then the linear system $6 H-\sum E_{i}$ embeds $Y$ as a smooth aCM surface $X$ of degree 21. The companion surface $X^{\prime}$ is not linearly normal embedded into $\mathbb{P}^{11}$.

This result is proved in Proposition 4.5 and Remarks 4.6 and 4.7. A very surprising feature of this example is that the companion surface $X^{\prime \prime}$ of $X^{\prime}$ is not $Y$ again but it is rather its projection.

Finally, we study companion surfaces related to Fermat arrangements. The $B_{3}$ configuration belongs also to this family. Our main results in this direction are Theorem 5.6, Proposition 5.7 and Remark 5.8. The precise statements are slightly too technical to quote them here.

We work over the field of complex numbers $\mathbb{C}$.

\section{Unexpected hypersurfaces}

The ground-breaking work [3] by Cook II, Harbourne, Migliore and Nagel introduced the concept of unexpected curves. This notion was generalized to arbitrary hypersurfaces in the subsequent article [17] by Harbourne, Migliore, Nagel and Teitler.

Definition 2.1. We say that a reduced set of points $Z \subset \mathbb{P}^{N}$ admits an unexpected hypersurface of degree $d$ if there exists a sequence of non-negative integers $m_{1}, \ldots, m_{s}$ such that for general points $P_{1}, \ldots, P_{s}$ the zero-dimensional subscheme $P=m_{1} P_{1}+\cdots+m_{s} P_{s}$ fails to impose independent conditions on forms of degree $d$ vanishing along $Z$ and the set of such forms is non-empty. In other words, we have

$h^{0}\left(\mathbb{P}^{N} ; \mathcal{O}_{\mathbb{P}^{N}}(d) \otimes I(Z) \otimes I(P)\right)>\max \left\{0, h^{0}\left(\mathbb{P}^{N} ; \mathcal{O}_{\mathbb{P}^{N}}(d) \otimes I(Z)\right)-\sum_{i=1}^{s}\left(\begin{array}{c}N+m_{i}-1 \\ N\end{array}\right)\right\}$.

Let us illustrate the above definition with an example.

Example 2.2. We consider the extended Fermat arrangement of planes in $\mathbb{P}^{3}$ :

$$
F=x y z w\left(x^{4}-y^{4}\right)\left(x^{4}-z^{4}\right)\left(x^{4}-w^{4}\right)\left(y^{4}-z^{4}\right)\left(y^{4}-w^{4}\right)\left(z^{4}-w^{4}\right)
$$


and we denote by $Z \subset \mathbb{P}^{3}$ the set of 28 points dual to the linear factors of $F$. We have:

$$
\begin{aligned}
I(Z)= & (x y z, x y w, x z w, y z w, \\
& \left.x y\left(x^{4}-y^{4}\right), x z\left(x^{4}-z^{4}\right), x w\left(x^{4}-w^{4}\right), y z\left(y^{4}-z^{4}\right), y w\left(y^{4}-w^{4}\right), z w\left(z^{4}-w^{4}\right)\right) .
\end{aligned}
$$

Using Macaulay2 [12], we easily check that there is one and only one unexpected surface of degree 6 containing $Z$ and three general points $P_{1}, P_{2}, P_{3}$ of multiplicities $m_{1}=m_{2}=$ $m_{3}=4$.

Following [2, Definition 2.5] we introduce also the following notion.

Definition 2.3 (Unexpected cone property). Let $Z$ be a finite set of points in $\mathbb{P}^{N}$ and let $d$ be a positive integer. We say that $Z$ has the unexpected cone property $\mathcal{C}(d)$, if for a general point $P \in \mathbb{P}^{N}$, there exists an unexpected (in the sense of Definition 2.1) hypersurface $S_{P}$ of degree $d$ and multiplicity $d$ at $P$ passing through all points in $Z$.

Example 2.4. We consider the set $Z \subset \mathbb{P}^{3}$ of 28 points introduced in Example 2.2. Using again Macaulay2 [12], we check that $Z$ has the unexpected cone property $\mathcal{C}(6)$ since for a general point $P \in \mathbb{P}^{3}$, there exists an unexpected surface $S_{P} \subset \mathbb{P}^{3}$ of degree 6 and multiplicity 6 at $P$ passing through all 28 points in $Z$.

\section{The BMSS duality}

For the sake of completeness let us explain what we understand for BMSS duality a notion which comes from [1] and [17, Section 4].

We consider integers $0<m \leq d \in \mathbb{Z}$, a set $Z \subset \mathbb{P}^{N}$ of points and a general point $P=\left(a_{0}: a_{1}: \cdots: a_{N}\right) \in \mathbb{P}^{N}$. We assume that there is a unique hypersurface $H_{Z} \subset \mathbb{P}^{N}$ of degree $d$ containing $Z$ and having multiplicity $m$ at $P$. The hypersurface $H_{Z}$ is defined by a bihomogeneous form

$$
F_{Z}(\underline{y}, \underline{x}):=F_{Z}\left(\left(y_{0}: y_{1}: \cdots: y_{N}\right),\left(x_{0}: x_{1}: \cdots: x_{N}\right)\right) \in \mathbb{C}[\underline{y}, \underline{x}] .
$$

of bidegree $(t, d)$ with $t \geq m$ (see [17, Lemma 3.1(d)]).

Note that the form must be bihomogeneous since if it were not, changing the projective coordinates of each of the two set of variables, the form would vary. Moreover, under mild hypotheses on $Z$ the bidegree of $F_{Z}(\underline{y}, \underline{x})$ is $(m, d)$ i.e. $d=m$ (see, for instance, [7, Proposition 1] for the general case and [17, Section 4] for special cases where much more can be said). It is important to point out that the bidegree of $F_{Z}(y, \underline{x})$ is not always $(m, d)$. In fact, in [1, Theorem 6] the authors considered a set $W \subset \mathbb{P}^{3}$ of 31 points with an expected quartic surface $S_{W} \subset \mathbb{P}^{3}$ having a general point $Q \in \mathbb{P}^{3}$ of multiplicity 3 and defined by a bihomogeneous polynomial $F_{W}(y, \underline{x})$ of bidegree $(5,4)$.

The BMSS duality allows us to view the bihomogeneous polynomial $F_{Z}(\underline{y}, \underline{x}) \in \mathbb{C}[\underline{y}][\underline{x}]$ of bidegree $(d, m)$ as a polynomial of degree $m$ in the indeterminates $\underline{x}$ with coefficients the point $P \in \mathbb{P}^{N}$ of multiplicity $m$, i.e., a family of hypersurfaces in the variables $\underline{x}$ parameterized by $P$ (the unexpected hypersurface):

$$
F_{Z, P}(\underline{x}):=F_{Z}\left(\left(a_{0}: \cdots: a_{N}\right), \underline{x}\right) \in \mathbb{C}\left[a_{0}, \ldots, a_{N}\right][\underline{x}]
$$

or, as a homogeneous polynomial of degree $d \geq m$ in the variables $\underline{y}$ with coefficients a point $Q=\left(b_{0}: \cdots: b_{N}\right) \in \mathbb{P}^{N}$, i.e. a family of hypersurfaces in the variables $\underline{y}$ parameterized by $Q$ :

$$
F_{Z, Q}(\underline{y}):=F_{Z}\left(\underline{y},\left(b_{0}: \cdots: b_{N}\right)\right) \in \mathbb{C}\left[b_{0}, \ldots, b_{N}\right][\underline{y}] .
$$

The BMSS duality establishes when the following assertions are true: 
- The tangent cone of $F_{Z, P}(\underline{x})$ at $Q$ coincides with the tangent cone of $F_{Z, Q}(\underline{y})$ at $P$.

- $F_{Z, P}(\underline{x})$ has multiplicity $m$ at a general point $Q$ and $F_{Z, Q}(\underline{y})$ has also multiplicity $m$ at a general point $P$.

In next section we discuss in detail an example which comes from the free arrangement of planes in $\mathbb{P}^{3}$ determined by linear factors of:

$x y z(x+y)(x-y)(x+z)(x-z)(x+w)(x-w)(y+z)(y-z)(y+w)(y-w)(z+w)(z-w)$

as well as an example which comes from the root system $H_{3}$.

\section{Companion varieties for root systems}

In [17, Section 3] the authors study unexpected hypersurfaces related to root systems. These are finite sets of vectors in a vector spaces satisfying certain number of conditions, see [18] for introduction, motivation and basic properties of this important notion. For us root systems are just a source of interesting configurations of points in projective spaces and we don't use explicitly any of many properties they enjoy. A vector $v$ in the root system in the vector space $V$ determines a point in the projective space $\mathbb{P}(V)$, which corresponds to the line spanned by $v$. As the set $Z$ one takes all points in $\mathbb{P}(V)$ determined in this way by all vectors in the root system.

The study in [17] was motivated by [5], where the first example of an unexpected curve coming from the $B_{3}$ root system was found. Computer experiments carried out in [17] provide the following list of root systems admitting a unique unexpected hypersurface. In Table 1 the number $N$ stands for the dimension of the ambient projective space $\mathbb{P}^{N}$ the number $d$ for the degree of an unexpected hypersurface and $m$ for the multiplicity in the general point.

\begin{tabular}{c|c|c|c} 
root system & $\mathrm{N}$ & $\mathrm{d}$ & $\mathrm{m}$ \\
\hline$B_{3}$ & 2 & 4 & 3 \\
\hline$B_{4}$ & 3 & 4 & 4 \\
\hline$D_{4}$ & 3 & 3 & 3 \\
\hline$F_{4}$ & 3 & 4 & 4 \\
\hline$H_{3}$ & 2 & 6 & 5 \\
\hline$H_{4}$ & 3 & 6 & 6
\end{tabular}

Table 1: Root systems determining a unique unexpected hypersurface

As [17, Section 3] and [1, Section 2] study the $B_{3}$ case in detail, we turn our attention to the $B_{4}$ root system and to the $H_{3}$ root system. We will encounter a couple of new phenomena in the course:

- in the case of the $B_{4}$ root system, after passing to the appropriate subspace of $\left[I\left(B_{4}\right)\right]_{4}$, we obtain a variety with an isomorphic companion variety;

- in the case of the $H_{3}$ root system we obtain the companion surface which is not aCM and which is embedded in a space of lower dimension than the initial variety.

\subsection{The $B_{4}$ root system}

The set $Z\left(B_{4}\right)$ consists of 16 points, which can be assigned the following coordinates:

$$
P_{1}=[1: 0: 0: 0] \quad P_{2}=[0: 1: 0: 0] \quad P_{3}=[0: 0: 1: 0] \quad P_{4}=[0: 0: 0: 1]
$$




$$
\begin{array}{ccc}
P_{5}=[1: 1: 0: 0] & P_{6}=[1: 0: 1: 0] & P_{7}=[1: 0: 0: 1] \\
P_{8}=[0: 1: 1: 0] & P_{9}=[0: 1: 0: 1] & P_{10}=[0: 0: 1: 1] \\
P_{11}=[1:-1: 0: 0] & P_{12}=[1: 0:-1: 0] & P_{13}=[1: 0: 0:-1] \\
P_{14}=[0: 1:-1: 0] & P_{15}=[0: 1: 0:-1] & P_{16}=[0: 0: 1:-1]
\end{array}
$$

The saturated ideal $I\left(B_{4}\right)$ is generated by

$x y z, x y w, x z w, y z w$,

and

$$
x y\left(x^{2}-y^{2}\right), x z\left(x^{2}-z^{2}\right), x w\left(x^{2}-w^{2}\right), y z\left(y^{2}-z^{2}\right), y w\left(y^{2}-w^{2}\right), z w\left(z^{2}-w^{2}\right) .
$$

A basis for the vector space $\left[I\left(B_{4}\right)\right]_{4}$ is given by including with the degree 4 elements listed in (3), the following elements:

$$
x y z w, x^{2} y z, x y^{2} z, x y z^{2}, x^{2} y w, x y^{2} w, x y w^{2}, x^{2} z w, x z^{2} w, x z w^{2}, y^{2} z w, y z^{2} w, y z w^{2} .
$$

The unexpected cone, written down in terms of this basis has then the following equation

$$
\begin{aligned}
F= & 3 a d\left(c^{2}-b^{2}\right) x^{2} y z+3 b d\left(a^{2}-c^{2}\right) x y^{2} z+3 c d\left(b^{2}-a^{2}\right) x y z^{2}+ \\
& 3 a c\left(b^{2}-d^{2}\right) x^{2} y w+3 b c\left(d^{2}-a^{2}\right) x y^{2} w+3 d c\left(a^{2}-b^{2}\right) x y w^{2}+ \\
& 3 a b\left(d^{2}-c^{2}\right) x^{2} z w+3 c b\left(a^{2}-d^{2}\right) x z^{2} w+3 d b\left(c^{2}-a^{2}\right) x z w^{2}+ \\
& 3 b a\left(c^{2}-d^{2}\right) y^{2} z w+3 c a\left(d^{2}-b^{2}\right) y z^{2} w+3 d a\left(b^{2}-c^{2}\right) y z w^{2}+ \\
& c d\left(d^{2}-c^{2}\right) x y\left(x^{2}-y^{2}\right)+b d\left(b^{2}-d^{2}\right) x z\left(x^{2}-z^{2}\right)+b c\left(c^{2}-b^{2}\right) x w\left(x^{2}-w^{2}\right)+ \\
& a d\left(d^{2}-a^{2}\right) y z\left(y^{2}-z^{2}\right)+a c\left(a^{2}-c^{2}\right) y w\left(y^{2}-w^{2}\right)+a b\left(b^{2}-a^{2}\right) z w\left(z^{2}-w^{2}\right) .
\end{aligned}
$$

It is immediately clear, that the generator $x y z w$ is not involved in the equation. The second property we observe is also quite surprising.

Proposition 4.1. All unexpected cones for the $B_{4}$ root system, have in their base locus 8 additional points:

$$
\begin{array}{llll}
Q_{1}=[1: 1: 1: 1] & Q_{2}=[1: 1: 1:-1] & Q_{3}=[1: 1:-1: 1] \\
Q_{4}=[1: 1:-1:-1] & Q_{5}=[1:-1: 1: 1] & & Q_{6}=[1:-1: 1:-1] \\
Q_{7}=[1:-1:-1: 1] & Q_{8}=[1:-1:-1:-1] . & &
\end{array}
$$

Proof. The vanishing of $F$ at the given points is easy to check. That the base locus is not larger can be verified by computing equations of unexpected cones for some specific values of $a, b, c, d$.

The points in $B_{4}$ together with the points $Q_{1}, \ldots, Q_{8}$ give the root system $F_{4}$.

Corollary 4.2. The unexpected cones for $B_{4}$ and $F_{4}$ are the same.

This observation has interesting implications for the companion threefolds. First, let us note, that a basis for $\left[I\left(F_{4}\right)\right]_{4}$ is given by the following forms:

$$
\begin{array}{lll}
m_{0}=x y\left(x^{2}-y^{2}\right), & m_{1}=x y\left(z^{2}-w^{2}\right), & m_{2}=x z\left(x^{2}-z^{2}\right), \\
m_{3}=x z\left(y^{2}-w^{2}\right), & m_{4}=x w\left(x^{2}-w^{2}\right), & m_{5}=x w\left(z^{2}-y^{2}\right), \\
m_{6}=y z\left(y^{2}-z^{2}\right), & m_{7}=y z\left(x^{2}-w^{2}\right), & m_{8}=y w\left(y^{2}-w^{2}\right) \\
m_{9}=y w\left(x^{2}-z^{2}\right), & m_{10}=z w\left(z^{2}-w^{2}\right), & m_{11}=z w\left(x^{2}-y^{2}\right) .
\end{array}
$$


Using these generators the equation (4) becomes

$$
\begin{aligned}
F= & c d\left(d^{2}-c^{2}\right) x y\left(x^{2}-y^{2}\right)+3 c d\left(b^{2}-a^{2}\right) x y\left(z^{2}-w^{2}\right)+b d\left(b^{2}-d^{2}\right) x z\left(x^{2}-z^{2}\right)+ \\
& 3 b d\left(a^{2}-c^{2}\right) x z\left(y^{2}-w^{2}\right)+b c\left(c^{2}-b^{2}\right) x w\left(x^{2}-w^{2}\right)+3 b c\left(a^{2}-d^{2}\right) x w\left(z^{2}-y^{2}\right)+ \\
& a d\left(d^{2}-a^{2}\right) y z\left(y^{2}-z^{2}\right)+3 a d\left(c^{2}-b^{2}\right) y z\left(x^{2}-w^{2}\right)+a c\left(a^{2}-c^{2}\right) y w\left(y^{2}-w^{2}\right)+ \\
& 3 a c\left(b^{2}-d^{2}\right) y w\left(x^{2}-z^{2}\right)+a b\left(b^{2}-a^{2}\right) z w\left(z^{2}-w^{2}\right)+3 a b\left(d^{2}-c^{2}\right) z w\left(x^{2}-y^{2}\right) .
\end{aligned}
$$

Let $\varphi: \mathbb{P}^{3} \rightarrow \mathbb{P}^{11}$ be the rational map defined by the generators in (5).

Proposition 4.3. The image of $\varphi$ is a smooth threefold of degree 40.

Proof. Let $\sigma: X \rightarrow \mathbb{P}^{3}$ be the simultaneous blow up of each of 24 points in $F_{4}$ with the exceptional divisor $\mathbb{E}$ (which splits into 24 projective planes, one over each of the points blown up) and as usual let $H=\sigma^{*} \mathcal{O}_{\mathbb{P}^{3}}(1)$. Since $\left\{p \in \mathbb{P}^{3} \mid m_{i}(p)=0\right.$ for $\left.i=0, \ldots, 11\right\}=$ $\left\{P_{1}, \ldots, P_{16}, Q_{1}, \ldots, Q_{8}\right\}$ and since the forms $m_{i}$ are unions of planes which at each base point $p$ have no common tangent, the linear system $L=4 H-\mathbb{E}$ is base point free and it defines a morphism onto its image $\varphi_{L}: X \rightarrow \mathbb{P}^{11}$ which lifts the map

$$
\varphi: \mathbb{P}^{3} \rightarrow \mathbb{P}^{11} \quad(x: y: z: w) \mapsto\left(m_{0}: m_{1}: \cdots: m_{11}\right) .
$$

Therefore, we have the commutative diagram

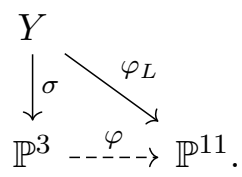

Let us call $X$ the image of $\varphi$. To check that the map $\varphi: \mathbb{P}^{3} \rightarrow Y$ has degree 1 and even more it is a birational map onto its image, we use the packages "Cremona" ([24], [25]) and "RationalMaps" [23] of Macaulay2 [12]. In fact, we have the following code block:

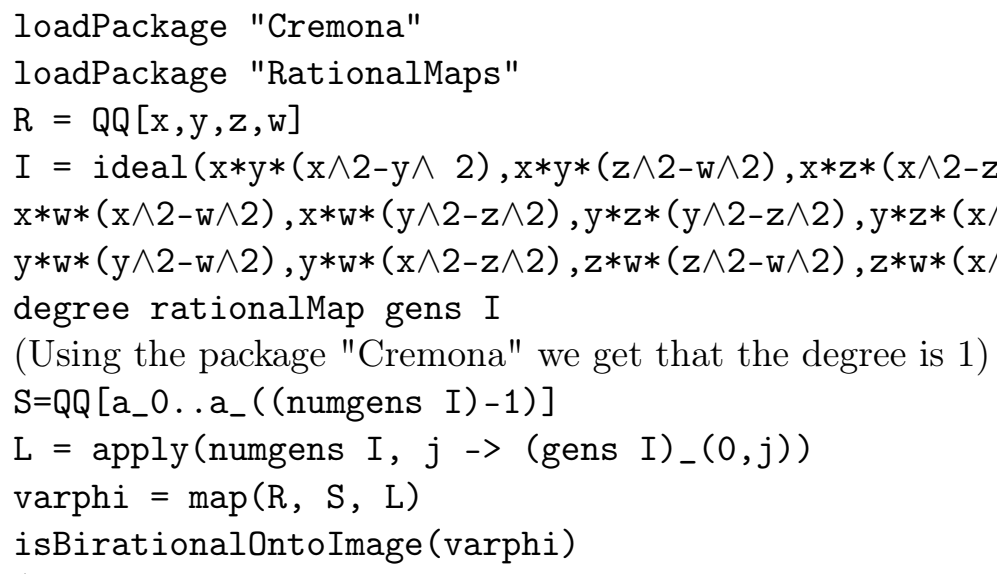

(Using the package "RationalMaps" we get that the map is birational onto its image.)

Once we know that $\varphi$ is a birational map onto its image we compute the degree of $Y$ : degree $(X)=L^{3}$. The smoothness of $X$ follows from the Jacobian criterion and a straightforward computation which shows that outside $\left\{P_{1}, \ldots, P_{16}, Q_{1}, \ldots, Q_{8}\right\}$ the rank of $\left(\begin{array}{llll}\frac{\partial m_{i}}{\partial x} & \frac{\partial m_{i}}{\partial y} & \frac{\partial m_{i}}{\partial z} & \frac{\partial m_{i}}{\partial w}\end{array}\right)_{i=0, \ldots, 11}$ is 4 .

It is worthwhile to point out that the Jacobian dual criterion as stated in [6, Theorem 2.18 and 3.2] or [21, Theorem 1.4] gives us a computer free proof of the birationality of $\varphi$. In fact, the ideal $I=\left(m_{0}, m_{1}, \ldots, m_{11}\right) \subset R:=\mathbb{C}[x, y, z, w]$ has a linear presentation:

$$
\cdots \longrightarrow R(-5)^{16} \stackrel{\psi}{\rightarrow} R(-4)^{12} \longrightarrow R \longrightarrow R / I \longrightarrow 0
$$


with

$$
\psi=\left(\begin{array}{cccccccccccccccc}
-z & 0 & 0 & 0 & 0 & 0 & -w & 0 & 0 & 0 & 0 & 0 & 0 & 0 & 0 & 0 \\
y & -y & 0 & 0 & 0 & 0 & 0 & 0 & 0 & 0 & -w & 0 & 0 & 0 & 0 & 0 \\
0 & x & -y & 0 & -z & 0 & 0 & 0 & 0 & 0 & 0 & 0 & -w & 0 & 0 & 0 \\
-y & 0 & x & -y & 0 & -z & 0 & 0 & 0 & 0 & 0 & w & 0 & 0 & -w & 0 \\
0 & 0 & 0 & x & 0 & 0 & 0 & 0 & 0 & 0 & 0 & 0 & 0 & w & 0 & -w \\
z & -z & 0 & z & x & y & -w & w & 0 & -w & 0 & 0 & 0 & 0 & 0 & 0 \\
0 & 0 & 0 & 0 & 0 & 0 & y & -y & 0 & 0 & z & -z & 0 & 0 & 0 & 0 \\
0 & 0 & 0 & 0 & w & 0 & 0 & x & -y & 0 & 0 & 0 & z & -z & 0 & 0 \\
0 & 0 & 0 & 0 & 0 & w & -y & 0 & x & -y & 0 & 0 & 0 & 0 & z & 0 \\
0 & 0 & 0 & 0 & 0 & 0 & 0 & 0 & 0 & x & 0 & 0 & 0 & 0 & 0 & z \\
0 & 0 & t & 0 & 0 & 0 & 0 & 0 & z & 0 & 0 & x & 0 & y & 0 & 0 \\
0 & 0 & 0 & 0 & 0 & 0 & 0 & 0 & 0 & 0 & -x & 0 & y & 0 & x & -y
\end{array}\right)
$$

Since $I$ has a linear presentation and $\operatorname{rank}(\psi)$ attains its maximal possible value, we apply the Jacobian dual criterion and we conclude that $\varphi$ is a birational map onto its image.

Remark 4.4. It follows from the equation (6) of the unexpected cone that $Y=i m(\varphi) \subset$ $\mathbb{P}^{11}$ is selfdual under the BMSS duality. This turns out to be true in a much more general set up. Indeed, let $Z \subset \mathbb{P}^{n}$ be a finite set of points and let $S_{P} \subset P^{n}$ be an unexpected hypersurface of degree $d$ and multiplicity $d$ at a general point $P=\left(a_{0}: \cdots: a_{n}\right) \in \mathbb{P}^{n}$ passing through all points in $Z$. Consider

$$
0=F_{Z}\left(\left(a_{0}: \cdots: a_{n}\right),\left(x_{0}: \cdots: x_{n}\right)\right)=\sum_{i=0}^{N} q_{i}\left(a_{0}: \cdots: a_{n}\right) f_{i}\left(x_{0}: \cdots: x_{n}\right)
$$

the equation of the unexpected cone $S_{P}$. Set $X=i m(\varphi)$ being $\varphi: \mathbb{P}^{n} \rightarrow \mathbb{P}^{N}$ the rational map defined by $f_{0}, \ldots, f_{N}$. By [7, Theorem 8$], X$ is selfdual under the BMSS duality.

\subsection{The $H_{3}$ root system}

Associated to the root system $H_{3}$ we have a set $Z\left(H_{3}\right)$ of 15 points, which can be assigned the following coordinates:

$$
\begin{aligned}
& P_{1}=[1: 0: 0] \quad P_{2}=[0: 1: 0] \quad P_{3}=[0: 0: 1] \\
& P_{4}=\left[1: u: u^{2}\right] \quad P_{5}=\left[-1: u: u^{2}\right] \quad P_{6}=\left[1:-u: u^{2}\right] \quad P_{7}=\left[1: u:-u^{2}\right] \\
& P_{8}=\left[u:-u^{2}: 1\right] \quad P_{9}=\left[-u: u^{2}: 1\right] \quad P_{10}=\left[u: u^{2}:-1\right] \quad P_{11}=\left[u: u^{2}: 1\right] \\
& P_{12}=\left[u^{2}: 1:-u\right] \quad P_{13}=\left[u^{2}:-1: u\right] \quad P_{14}=\left[-u^{2}: 1: u\right] \quad P_{15}=\left[u^{2}: 1: u\right]
\end{aligned}
$$

where $u^{2}-u-1=0$, so that $u$ is the golden ratio or its Galois conjugate $\bar{u}=1-u$. The configuration together with 6 lines, each of them containing 5 configuration points $(2$ of them are at the infinity) is visualised at Figure 1 . The 10 thin lines contain 3 configuration points each. In order to increase the readability of the figure, only numbers of points are indicated.

The lines with 5 configuration points have rather simple equations:

$$
\begin{gathered}
L_{1}: y-(u-1) z=0, \quad L_{2}: y+(u-1) z=0, \quad L_{3}: x-u z=0, \quad L_{4}: x+u z=0, \\
L_{5}: x-(u-1) y=0, \quad L_{6}: x+(u-1) y=0 .
\end{gathered}
$$




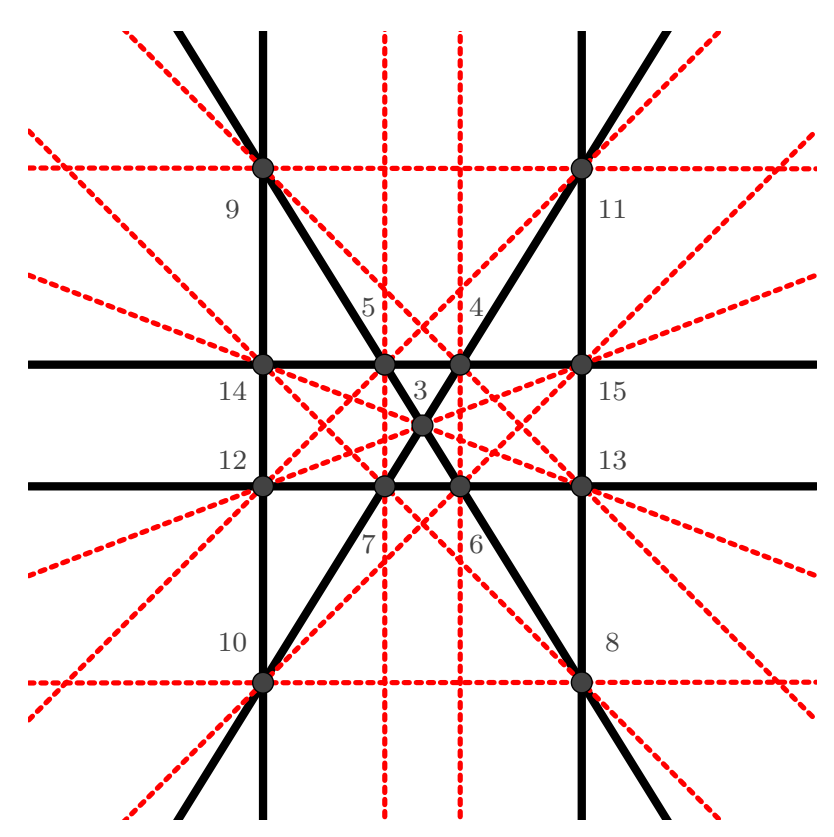

Figure 1: The $H_{3}$ configuration of points with $P_{1}$ and $P_{2}$ at the infinity

The saturated ideal $I\left(H_{3}\right)$ is generated by six quintics. One can choose as the generators the products of all but one line:

$$
\begin{aligned}
& f_{1}=L_{2} L_{3} L_{4} L_{5} L_{6}, f_{2}=L_{1} L_{3} L_{4} L_{5} L_{6}, f_{3}=L_{1} L_{2} L_{4} L_{5} L_{6}, \\
& f_{4}=L_{1} L_{2} L_{3} L_{5} L_{6}, f_{5}=L_{1} L_{2} L_{3} L_{4} L_{6}, f_{6}=L_{1} L_{2} L_{3} L_{4} L_{5} .
\end{aligned}
$$

This follows from the fact that the lines $L_{1}, \ldots, L_{6}$ form the so called star-configuration, see [11, Proposition 2.9], see also [22, Lemma 3.1 and 3.2] for an alternative argument. Below we present an equivalent direct argument based on the Hilbert-Burch theorem.

The ideal $I\left(H_{3}\right)$ has 13 generators, all in degree 6 . Indeed, $I\left(H_{3}\right)$ is generated by the 6 maximal minors of the $6 \times 5$ matrix $U$ with linear entries:

$$
U=\left(\begin{array}{ccccc}
L_{1} & 0 & 0 & 0 & 0 \\
-L_{2} & L_{2} & 0 & 0 & 0 \\
0 & -L_{3} & L_{3} & 0 & 0 \\
0 & 0 & -L_{4} & L_{4} & 0 \\
0 & 0 & 0 & -L_{5} & L_{5} \\
0 & 0 & 0 & 0 & -L_{6}
\end{array}\right)
$$

Therefore, $I\left(H_{3}\right)$ has a minimal free resolution:

$$
0 \longrightarrow S(-6)^{5} \stackrel{U}{\rightarrow} S(-5)^{6} \longrightarrow I\left(H_{3}\right) \longrightarrow 0
$$

with $S=\mathbb{C}[x, y, z]$ and we conclude that $\operatorname{dim} I\left(H_{3}\right)_{6}=13$. It is convenient to write down explicitly generators of $I\left(H_{3}\right)_{6}$ :

$$
g_{i}=L_{1} f_{i}, \text { for } i=1, \ldots, 6,
$$

$g_{7}=L_{2} f_{1}, g_{8}=L_{2} f_{3}, g_{9}=L_{2} f_{4}, g_{10}=L_{2} f_{5}, g_{11}=L_{2} f_{6}, g_{12}=L_{3} f_{1}, g_{13}=L_{3} f_{2}$.

The set of points $Z\left(H_{3}\right)$ admits a unique unexpected curve $C \subset \mathbb{P}^{2}$ of degree 6 and multiplicity 5 at a general point $P=(a: b: c) \in \mathbb{P}^{2}$ defined by the following equation:

$$
F_{Z\left(H_{3}\right)}((a: b: c),(x: y: z))=\sum_{i=1}^{13} h_{i}(a: b: c) g_{i}(x: y: z),
$$


where

$$
\begin{aligned}
& h_{1}=\left(\frac{7}{2} u+2\right) \cdot b \cdot\left(b^{4}+(-44 u+72) a b^{2} c+(-6 u+8) b^{2} c^{2}+(-4 u+12) a c^{3}+(u-3) c^{4}\right), \\
& h_{2}=\frac{-10 u-5}{4}\left(a+\frac{u+2}{5} b+\frac{-3 u-1}{5} c\right)(b+(u-1) c)^{4}, \\
& h_{3}=\frac{2 u-1}{4}(a+(u+2) b+(u-1) c)(a-u c)^{4} \text {, } \\
& h_{4}=\frac{-2 u+1}{4}(a-(u+2) b-(u-1) c)(a+u c)^{4}, \\
& h_{5}=\frac{4 u+3}{4}(a-(u-1) b)^{4}(a+u b-(u-3) c), \\
& h_{6}=\frac{-4 u-3}{4}(a+(u-1) b)^{4}(a-u b+(u-3) c), \\
& h_{7}=\frac{10 u+5}{4}\left(a+\frac{-u-2}{5} b+\frac{-3 u-1}{5} c\right)(b-(u-1) c)^{4}, \\
& h_{8}=\frac{2 u-1}{4}(a-(u+2) b+(u-1) c)(a-u c)^{4}, \\
& h_{9}=\frac{-2 u+1}{4}(a+(u+2) b-(u-1) c)(a+u c)^{4} \text {, } \\
& h_{10}=\frac{-4 u-3}{4}(a-(u-1) b)^{4}(a+u b+(u-3) c) \text {, } \\
& h_{11}=\frac{4 u+3}{4}(a+(u-1) b)^{4}(a-u b-(u-3) c) \text {, } \\
& h_{12}=\frac{-3 u-1}{2}(b+u c)(b-(u-1) c)^{4}, \\
& h_{13}=\frac{3 u+1}{2}(b-u c)(b+(u-1) c)^{4} \text {. } \\
& \text { ( }
\end{aligned}
$$

Figure 2: Unexpected sextic with a point of multiplicity 5

Figure 2 indicates the unexpected sextic curve with multiplicity 5 at a general point. The configuration points of the $\mathrm{H}_{3}$ root system are arranged as in Figure 1. The lines through only 3 configuration points and the names of the points are omitted for transparency.

Let $\varphi: \mathbb{P}^{2} \rightarrow \mathbb{P}^{12}$ be the rational map defined by the 13 generators $g_{1}, \ldots, g_{13}$ of $I\left(Z\left(H_{3}\right)\right)$. 
Proposition 4.5. The image of $\varphi$ is an arithmetically Cohen-Macaulay smooth surface $X \subset \mathbb{P}^{12}$ of degree 21 whose homogeneous ideal $I(X)$ is generated by quadrics.

Proof. We consider $\pi: Y \rightarrow \mathbb{P}^{2}$ the blow up of $\mathbb{P}^{2}$ at the 15 points of $Z\left(H_{3}\right)$. Set $H=$ $\pi^{*} \mathcal{O}_{\mathbb{P}^{2}}(1)$ and denote by $E_{i}, i=1, \ldots, 15$, the exceptional divisors.

Claim 1: The linear system $L=6 H-\sum_{i=1}^{15} E_{i}$ is base point free and very ample. Proof of Claim 1. We sheafify the exact sequence (8) and we get the exact sequence:

$$
0 \longrightarrow \mathcal{O}_{\mathbb{P}^{2}}(-6)^{5} \longrightarrow \mathcal{O}_{\mathbb{P}^{2}}(-5)^{6} \longrightarrow \mathcal{I}_{Z\left(H_{3}\right)} \longrightarrow 0 .
$$

We compute the cohomology and we get $H^{1}\left(\mathcal{I}_{Z\left(H_{3}\right)}(5)\right)=0$. Therefore, the linear system $L=6 H-\sum_{i=1}^{15} E_{i}$ is base point free (see [4, Theorem 1.4]). Moreover, since no line in $\mathbb{P}^{2}$ contains 6 points of $Z\left(H_{3}\right)$, we can apply [4, Theorem 3.1] and we conclude that $L$ is very ample.

Since $L=6 H-\sum_{i=1}^{15} E_{i}$ is base point free and very ample, it defines an embedding, i.e. an isomorphism onto its image $\varphi_{L}: Y \rightarrow \mathbb{P}^{12}$ which lifts the rational map $\varphi: \mathbb{P}^{2} \rightarrow \mathbb{P}^{12}$. Therefore, we have the commutative diagram

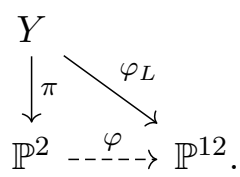

and the image of $\varphi$ is a smooth surface $X \subset \mathbb{P}^{12}$ of degree $L^{2}=21$.

Let us now prove that $X$ is an arithmetically Cohen-Macaulay surface. First we compute the $h$-vector $\left(h_{Z\left(H_{3}\right)}(i)\right)$ of the set of points $Z\left(H_{3}\right) \subset \mathbb{P}^{2}$ where

$$
\begin{aligned}
h_{Z\left(H_{3}\right)}(i) & :=\Delta H\left(Z\left(H_{3}\right), i\right)=H\left(Z\left(H_{3}\right), i\right)-H\left(Z\left(H_{3}\right), i-1\right) \\
& =\operatorname{dim}\left(\frac{k[x, y, z]}{I\left(Z\left(H_{3}\right)\right)}\right)_{i}-\operatorname{dim}\left(\frac{k[x, y, z]}{I\left(Z\left(H_{3}\right)\right)}\right)_{i-1} .
\end{aligned}
$$

The $h$-vector of $Z\left(H_{3}\right)$ is $(1,2,3,4,5)$. Therefore, $h_{Z\left(H_{3}\right)}(i)=0$ for all $i \geq 5$ and $\sigma=5$, where $\sigma$ is the least integer $t$ such that $\Delta H\left(Z\left(H_{3}\right), t\right)=0$. By [10, Theorem B], $X$ is an arithmetically Cohen-Macaulay surface and by [10, Theorem $\mathrm{C}], I(X)$ is generated by quadrics which proves what we want.

Turning to the companion surface we take a closer look at the polynomials $h_{1}, h_{2}, \ldots, h_{13}$. First of all, it can be checked directly that $h_{1}$ is a linear combination of the remaining 12 polynomials. These polynomials, in turn, can be paired according to their fourth power factors. Removing the constant terms and taking linear combinations, we obtain the following 12 quintics in variables $(a: b: c)$ :

$$
\begin{gathered}
q_{1}=a(b-(u-1) c)^{4}, \quad q_{2}=(b+u c)(b-(u-1) c)^{4}, \\
q_{3}=a(b+(u-1) c)^{4}, \quad q_{4}=(b-u c)(b+(u-1) c)^{4}, \\
q_{5}=b(a-u c)^{4}, \quad q_{6}=(a+(u-1) c)(a-u c)^{4}, \\
q_{7}=b(a+u c)^{4}, \quad q_{8}=(a-(u-1) c)(a+u c)^{4}, \\
q_{9}=c(a-(u-1) b)^{4}, \quad q_{10}=(a+u b)(a-(u-1) b)^{4}, \\
q_{11}=c(a+(u-1) b)^{4}, \quad q_{12}=(a-u b)(a+(u-1) b)^{4} .
\end{gathered}
$$


Interestingly, the unexpected curve written down in these generators has the following form

$$
\begin{aligned}
F_{Z\left(H_{3}\right)} & =\frac{1}{2}\left[(3 u+1)\left[M_{1} f_{1} q_{1}-x f_{1} q_{2}-M_{2} f_{2} q_{3}+x f_{2} q_{4}\right]\right. \\
& +(1-2 u)\left[M_{3} f_{3} q_{5}-y f_{3} q_{6}-M_{4} f_{4} q_{7}+y f_{4} q_{8}\right] \\
& \left.+(3 u+1)\left[M_{5} f_{5} q_{9}-z f_{5} q_{10}-M_{6} f_{6} q_{11}+z f_{6} q_{12}\right]\right],
\end{aligned}
$$

where $M_{1}, \ldots, M_{6}$ are Galois conjugates of lines $L_{1}, \ldots, L_{6}$ and $f_{1}, \ldots, f_{6}$ are defined in (7):

$$
\begin{array}{ll}
M_{1}: y+u z=0, & M_{2}: y-u z=0, \\
M_{3}: x-\bar{u} z=0, & M_{4}: x+\bar{u} z=0, \\
M_{5}: x+u y=0, & M_{6}: x-u y=0 .
\end{array}
$$

These lines are indicated as dashed lines in Figure 3, the $L$-lines are indicated there as the thin lines. The dashed lines intersect in the Galois conjugate $H_{3}$ configuration of points. In particular the origin and the two points at the infinity belong to both configurations.

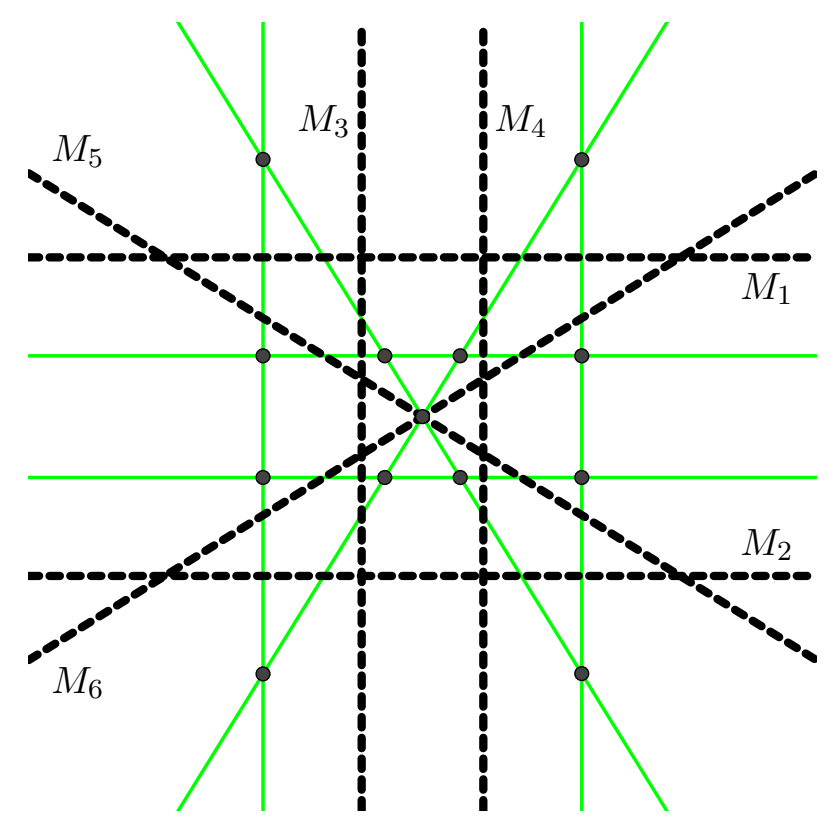

Figure 3: The $H_{3}$ configuration of points and the Galois conjugate lines

Remark 4.6. We describe the companion surface $X^{\prime}$ of $X$.

Let $\psi: \mathbb{P}^{2} \rightarrow \mathbb{P}^{11}$ be the rational map defined by the 12 generators $q_{1}, \ldots, q_{12}$ and we call $X^{\prime}$ the image of $\psi$. To check that the map $\psi: \mathbb{P}^{2} \rightarrow \mathbb{P}^{11}$ has degree one and even more it is a birational map onto its image we use the packages "Cremona" and "RationalMaps" of Macaulay2 as before.

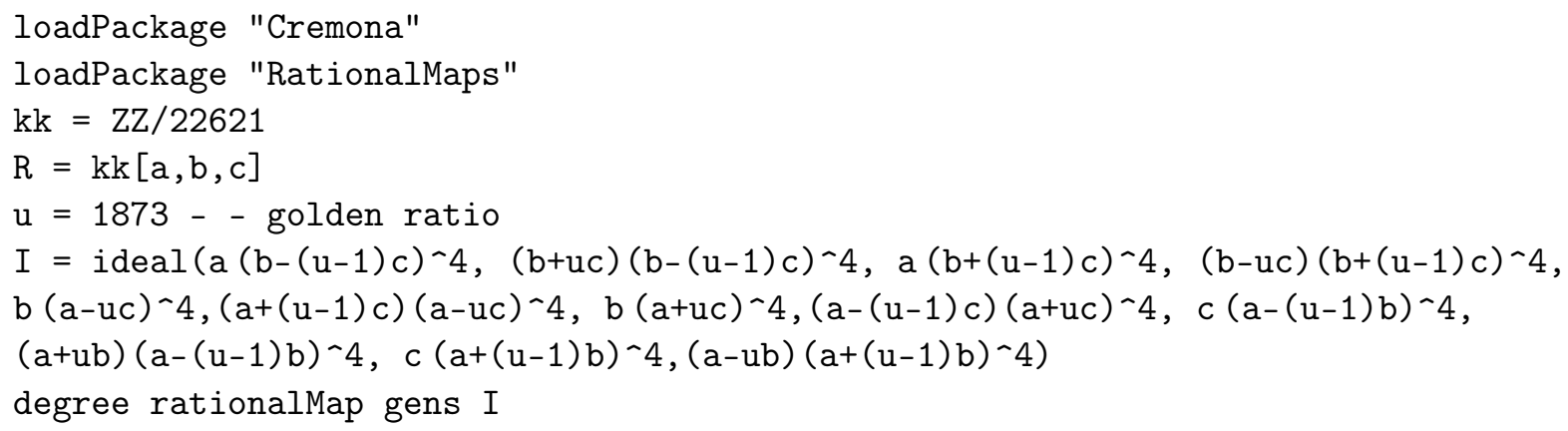


(Using the package "Cremona" we get that the degree is 1)

$\mathrm{S}=\mathrm{kk}\left[\mathrm{q}_{-} 1 \ldots \mathrm{q}_{-}\right.$(numgens I)]

varphi $=\operatorname{map}(R, S$, gens $I)$

isBirationalOntoImage(varphi) - - true

(Using the package "RationalMaps" we get that the map is birational onto its image)

Once we know that $\psi$ is a birational map onto its image we compute the degree of $X^{\prime}$, it is 25. The smoothness of $X^{\prime}$ follows from the Jacobian criterion and a computation which shows that the rank of $\left(\begin{array}{lll}\frac{\partial q_{i}}{\partial a} & \frac{\partial q_{i}}{\partial b} & \frac{\partial q_{i}}{\partial c}\end{array}\right)_{i=1, \ldots, 12}$ is 3 . It is a projection of the Veronese $V_{2,5}$ by a subspace of dimension eight that does not intersect the Secant variety. Moreover, the ideal of $X^{\prime}$ is generated by quadrics and cubics.

Remark 4.7. We consider the rational map $\bar{\varphi}: \mathbb{P}^{2} \rightarrow \mathbb{P}^{11}$ where

$$
\bar{\varphi}=\left(M_{1} f_{1}, x f_{1}, M_{2} f_{2}, x f_{2}, M_{3} f_{3}, y f_{3}, M_{4} f_{4}, y f_{4}, M_{5} f_{5}, z f_{5}, M_{6} f_{6}, z f_{6}\right)
$$

and we call $\bar{X}$ its image. Using Macaulay2 we get that $\bar{\varphi}$ is a birational map onto $\bar{X}$, degree $(\bar{X})=21$ and $I(\bar{X})$ is generated by 32 quadrics. The surface $\bar{X} \subset \mathbb{P}^{11}$ can be seen as a projection of the surface $X \subset \mathbb{P}^{12}$ described in Proposition 4.5. However, $\bar{X}$ is not aCM, as its $h$-vector is $h:(1,9,13,-3,1)$, so it is not positive.

\section{Companion surfaces for Fermat arrangements of lines}

Recall that in suitable coordinates the points in the root system $B_{3}$ are dual to linear factors of the Fermat-type polynomial

$$
F_{2,3}(x: y: z)=x y z\left(x^{2}-y^{2}\right)\left(y^{2}-z^{2}\right)\left(z^{2}-x^{2}\right) .
$$

Thus it is natural to generalize $B_{3}$ from the perspective of Fermat-type arrangements. We follow this path of thoughts in this section. Our notation is consistent with the notation introduced in [27].

Let

$$
F_{m, 0}(x: y: z)=\left(x^{m}-y^{m}\right)\left(y^{m}-z^{m}\right)\left(z^{m}-x^{m}\right)
$$

and let $Z\left(F_{m, 0}\right)$ be the set of points dual to the linear factors of $F_{m, 0}$. Thus $Z\left(F_{m, 0}\right)$ is a set of $3 \mathrm{~m}$ points distributed evenly on the coordinate lines. This is visualised in Figure $4 \mathrm{a}$ for $m=5$.

We recall a result $[27$, Theorem 4.6$]$ which motivated our present research.

Theorem 5.1 (Szpond). Let $m \geq 5$ be an integer and let $R=(a: b: c)$ be a general point in $\mathbb{P}^{2}$. The set $Z\left(F_{m .0}\right)$ admits a unique irreducible unexpected curve $C_{R, m}$ of degree $m+2$ and multiplicity $m+1$. Moreover, all these curves $C_{R, m}$ pass through the coordinate points.

Set $F_{m, 3}=x y z F_{m, 0}$. It turns out that for the augmented configuration

$$
Z\left(F_{m, 3}\right)=Z\left(F_{m, 0}\right) \cup(1: 0: 0) \cup(0: 1: 0) \cup(0: 0: 1)
$$

the curve $C_{R, m}$ is unexpected as soon as $m \geq 2$. For $m=2$ we get exactly the unexpected curve associated to the $B_{3}$ root system.

Proposition 5.2. The ideal $I\left(Z\left(F_{m, 3}\right)\right) \subset S=\mathbb{C}[x, y, z]$ is generated by

$$
x y z, G_{x}=y z\left(y^{m}-(-z)^{m}\right), G_{y}=z x\left(z^{m}-(-x)^{m}\right), G_{z}=x y\left(x^{m}-(-y)^{m}\right) .
$$




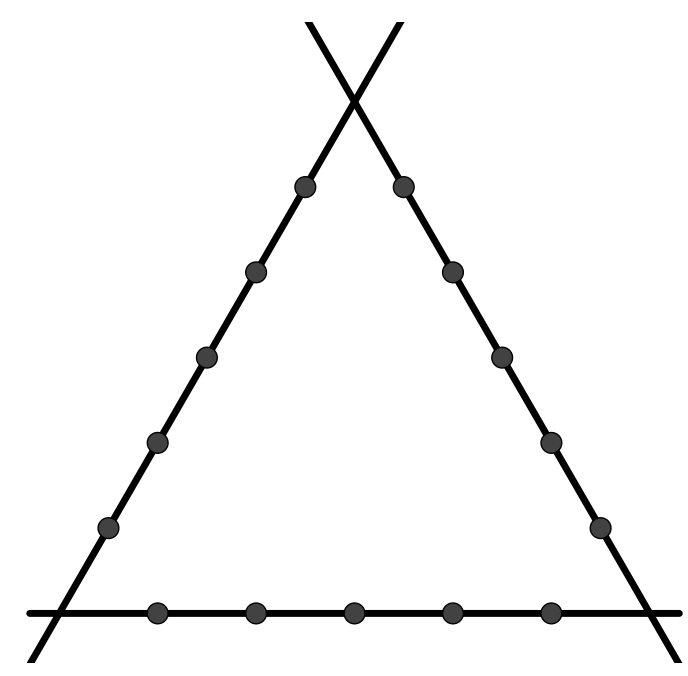

(a) The ordinary configuration

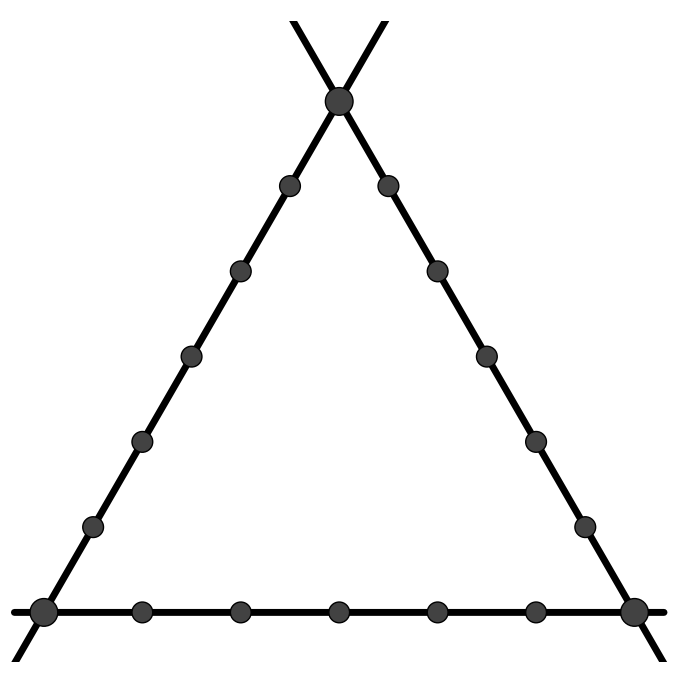

(b) The augmented configuration

Figure 4: Fermat configurations of points for $m=5$

Even more, $I\left(Z\left(F_{m, 3}\right)\right)$ is defined by the maximal minors of the matrix

$$
M=\left(\begin{array}{ccc}
y^{m}-(-z)^{m} & z^{m}-(-x)^{m} & x^{m}-(-y)^{m} \\
x & 0 & 0 \\
0 & y & 0 \\
0 & 0 & z
\end{array}\right)
$$

and, hence, it has a minimal free resolution of the following type:

$$
0 \longrightarrow S(-m-3)^{3} \stackrel{M}{\longrightarrow} S(-3) \oplus S(-m-2)^{3} \longrightarrow I\left(Z\left(F_{m, 3}\right)\right) \longrightarrow 0 .
$$

Proof. First we note that the reason for the sign in the three binomial generators depending on the parity of $m$ is due to the convention that a point $(p: q: r)$ is dual to the line $p x+q y+r z=0$. Thus, for example, if $m=3$ and $\varepsilon$ is a primitive root of the unity of order 3 , then

$$
(x-\varepsilon y)\left(x-\varepsilon^{2} y\right)(x-y)=x^{3}-y^{3},
$$

and the dual points are

$$
(1:-\varepsilon: 0),\left(1:-\varepsilon^{2}: 0\right),(1:-1: 0) .
$$

Their ideal is generated by $\left(z, x^{3}+y^{3}\right)$.

It is now easy to see that for arbitrary $m$ the trace of $Z\left(F_{m, 0}\right)$ on the coordinate line, say $x=0$, is a set of zeroes of a complete intersection ideal generated by

$$
x \text { and } y z\left(y^{m}+(-1)^{m+1} z^{m}\right)
$$

and similarly on the other lines. Thus

$$
\begin{aligned}
I\left(Z\left(F_{m, 3}\right)\right)= & (x y, y z, z x) \cap\left(x, y z\left(y^{m}+(-1)^{m+1} z^{m}\right)\right) \\
& \cap\left(y, x z\left(z^{m}+(-1)^{m+1} x^{m}\right)\right) \cap\left(z, x y\left(x^{m}+(-1)^{m+1} y^{m}\right)\right) .
\end{aligned}
$$

and the claim follows readily. 
We are interested in homogeneous elements $\left[I\left(Z\left(F_{m, 3}\right)\right)\right]_{m+2}$ in $I\left(Z\left(F_{m, 3}\right)\right)$ of degree $(m+2)$. They are $G_{x}, G_{y}, G_{z}$ and monomials of the form

$$
x y z \lambda \text { where } \lambda \text { is a monomial of degree } m-1 \text {. }
$$

Hence

$$
\operatorname{dim}\left[I\left(Z\left(F_{m, 3}\right)\right)\right]_{m+2}=\frac{1}{2} m^{2}+\frac{1}{2} m+3 .
$$

Geometrically the elements in $\left[I\left(Z\left(F_{m, 3}\right)\right)\right]_{m+2}$ correspond to elements of the linear system of forms of degree $m+2$ vanishing at all points of the set $Z\left(F_{m, 3}\right)$.

\subsection{Positivity on anticanonical surfaces}

Before we continue the study of the linear system determined by $\left[I\left(Z\left(F_{m, 3}\right)\right)\right]_{m+2}$, we need to recall some results on anticanonical systems on surfaces. Results presented here were obtained by Harbourne in [16] and [15]. We begin by the following useful Lemma, which is a combination of Lemma II.2 and Corollary II.3 in [15] and Lemma 2.2 and Corollary 2.3 in [16].

Lemma 5.3 (Harbourne). Let $Y$ be a smooth rational surface and let $D$ be a nef class on $Y$. Then

$$
h^{2}(Y, D)=0 \quad \text { and } \quad D^{2} \geq 0 .
$$

Moreover, if $-K_{Y}$ is effective, then so is $D$.

Assuming the effectivity of the anticanonical class, one can say in fact much more. The following statement is extracted from a much more precise (and complicated) result of Harbourne [15, Theorem III.1] and [16, Theorem 2.11].

Proposition 5.4 (Harbourne). Let $Y$ be a smooth anticanonical surface and let $D$ be a nef class on $Y$ such that $-K_{Y} . D \geq 2$. Then $D$ is non-special, i.e., $h^{1}(Y, D)=0$ and the linear system $|D|$ is base point free.

Thus the anticanonical degree at least 2 implies that $|D|$ defines a morphism. If this degree is at least 3 , then the positivity of $|D|$ increases, see [16, Proposition 3.2].

Proposition 5.5 (Harbourne). Let $Y$ be a smooth anticanonical surface and let $D$ be a nef class on $Y$ such that $-K_{Y} \cdot D \geq 3$ and $D^{2}>0$. Then the morphism $\varphi_{D}$ defined by the elements of $|D|$ is birational and its image $Y^{\prime}$ is a normal surface obtained by contracting all curves perpendicular to $|D|$. Moreover a general member of $|D|$ is smooth and irreducible.

\subsection{On the positivity of maps determined by Fermat arrangements}

Let $m$ be fixed and let $\varepsilon$ be a primitive root of the unity of order $m$. It is convenient to introduce the following notation:

$$
P_{x, \alpha}=\left(0: 1: \varepsilon^{\alpha}\right), P_{y, \alpha}=\left(1: 0: \varepsilon^{\alpha}\right), P_{z, \alpha}=\left(1: \varepsilon^{\alpha}: 0\right)
$$

for $\alpha=0, \ldots, m-1$ and

$$
P_{x, y}=(0: 0: 1), P_{x, z}=(0: 1: 0), P_{y, z}=(1: 0: 0) .
$$

Our next result concerns the positivity of the linear system corresponding to $\left[I\left(Z\left(F_{m, 3}\right)\right)\right]_{m+2}$. 
Theorem 5.6. Let $f: Y \rightarrow \mathbb{P}^{2}$ be the blow up at the points of the set $Z\left(F_{m, 3}\right)$ with the exceptional divisors $E_{a, \alpha}, E_{a, b}$ over the points $P_{a, \alpha}$ and $P_{a, b}$ respectively, where $a, b \in$ $\{x, y, z\}$ and $\alpha \in\{0, \ldots, m-1\}$. Let $H=f^{*} \mathcal{O}_{\mathbb{P}^{2}}(1)$ be the pull-back of the hyperplane bundle and let $\mathbb{E}$ be the union of all exceptional divisors of $f$. Then the linear system

$$
L=(m+2) H-\mathbb{E}
$$

is base point free and defines a morphism $\varphi_{L}$ birational onto its image

$$
\varphi_{L}: Y \rightarrow X \subset \mathbb{P}^{N}, \quad\left(N=\frac{1}{2}\left(m^{2}+m\right)+2\right),
$$

which is an isomorphism away from the proper transforms of the coordinate lines.

Proof. Let

$$
\begin{aligned}
& N_{x}=H-E_{x, y}-E_{x, z}-E_{x, 0}-\cdots-E_{x, m-1} \\
& N_{y}=H-E_{x, y}-E_{y, z}-E_{y, 0}-\cdots-E_{y, m-1} \\
& N_{z}=H-E_{x, z}-E_{y, z}-E_{z, 0}-\cdots-E_{z, m-1}
\end{aligned}
$$

be the proper transformations of the coordinate lines.

Our first claim is that $L$ is nef and the only curves having the intersection number with $L$ equal zero are $N_{x}, N_{y}$ and $N_{z}$.

It is obvious that $L . N_{a}=0$ for all $a \in\{x, y, z\}$, so it is enough to check that $L$ has positive intersection with all other irreducible curves on $Y$. Certainly it is $L . E=1$ for all exceptional divisors of $f$. Let $C$ be an irreducible plane curve of degree $d$, different from the coordinate lines, passing through the points $P_{a, \alpha}$ and $P_{a, b}$ with multiplicities $m_{a, \alpha}$ and $m_{a, b}$ respectively. Let

$$
\widetilde{C}=d H-\sum m_{a, \alpha} E_{a, \alpha}-\sum m_{a, b} E_{a, b}
$$

be the proper transform of $C$. Then

$$
\begin{aligned}
& 0 \leq \widetilde{C} \cdot N_{x}=d-m_{x, y}-m_{x, z}-m_{x, 0}-\cdots-m_{x, m-1}, \\
& 0 \leq \widetilde{C} \cdot N_{y}=d-m_{x, y}-m_{y, z}-m_{y, 0}-\cdots-m_{y, m-1}, \\
& 0 \leq \widetilde{C} \cdot N_{z}=d-m_{x, z}-m_{y, z}-m_{z, 0}-\cdots-m_{z, m-1} .
\end{aligned}
$$

Adding the inequalities (10), (11) and (12) and rearranging terms we get

$$
3 d-2\left(m_{x, y}+m_{x, z}+m_{y, z}\right)-\sum m_{a, \alpha} \geq 0 .
$$

Since

$$
M . \widetilde{C}=4 d-m_{x, y}-m_{x, z}-m_{y, z}-\sum m_{a, \alpha}
$$

we conclude that $M . \widetilde{C}>0$ as asserted.

For the rest of the proof, the key observation is that the surface $Y$ is an anticanonical surface, i.e., $-K_{Y}$ is an effective divisor. Indeed

$$
-K_{Y}=N_{x}+N_{y}+N_{z}+E_{x, y}+E_{x, z}+E_{y, z}
$$

Since

$$
-K_{Y} \cdot M=3
$$

Proposition 5.4 implies that $|M|$ is non-special and base point free. Revoking Lemma 5.3 we have thus the vanishing

$$
h^{1}(Y, L)=h^{2}(Y, D)=0,
$$


which together with the Riemann-Roch formula on $Y$ reconfirms the computation in (9). Since

$$
L^{2}=m^{2}+m+1>0,
$$

it follows from Proposition 5.5 that the morphism defined by $|L|$ is birational and its image is a normal surface of degree $m^{2}+m+1$ in $\mathbb{P}^{N}$ with $N=\frac{1}{2}\left(m^{2}+m\right)+2$ singular in exactly 3 points, which are images of the proper transforms of the coordinate lines in $\mathbb{P}^{2}$.

We now turn to the equations defining $X$. To this end let us be a little bit more specific about the coordinates of the rational map $\varphi$ determined by the commutative diagram

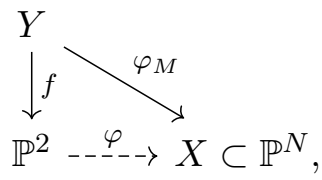

which is not just incidentally similar to that in Proposition 4.3. We can assume that

$$
\varphi=\left(x y z \mu_{d}: G_{x}: G_{y}: G_{z}\right),
$$

where $d=m-1$ and

$$
\mu_{d}: \mathbb{P}^{2} \hookrightarrow \mathbb{P}^{N-3}
$$

denotes the Veronese embedding of degree $d$ and the rational map $x y z \mu_{d}$ is given by coordinates of $\mu_{d}$ multiplied by the monomial $x y z$.

It is well known, see for example [28, Corollary 7.2.3], that ideal of the image $V_{d}$ of $\mu_{d}$ is generated by $\frac{(d-1) d(d+1)(d+6)}{8}$ quadratic binomials.

Consider the projection $\pi: \mathbb{P}^{N} \rightarrow \mathbb{P}^{N-3}$ determined by the plane $\Pi$ spanned by the last three coordinate points in $\mathbb{P}^{N}$. Then $\pi(X)=V_{d}$ and the mapping $\varphi$ (or equivalently $\left.\varphi_{L}\right)$ can be considered as the "unprojection" of the Veronese surface in $\mathbb{P}^{N-3}$. We use the word unprojection to indicate a morphism inverse to a projection, not quite in the sense as introduced to the birational geometry by Miles Reid.

Proposition 5.7. Let $m \geq 4$ be a fixed integer and let $I=I(X)$ be the ideal of $X \subset \mathbb{P}^{N}$. It holds:

(1) $X \subset \mathbb{P}^{N}$ is an aCM surface.

(2) The ideal $I$ is generated by forms of degree $\leq 3$. More precisely, $I$ is generated by $3\left(\begin{array}{c}m+2 \\ 4\end{array}\right)$ quadrics and one cubic $C_{m}$, where for $m$ odd

$$
C_{m}: 2 H_{x} H_{y} H_{z}+G_{x} H_{y} H_{z}+H_{x} G_{y} H_{z}+H_{x} H_{y} G_{z}-G_{x} G_{y} G_{z},
$$

and for $m$ even

$$
C_{m}: G_{x} H_{y} H_{z}+H_{x} G_{y} H_{z}+H_{x} H_{y} G_{z}-G_{x} G_{y} G_{z},
$$

where $H_{x}=x^{m} y z, H_{y}=x y^{m} z$ and $H_{z}=x y z^{m}$.

Proof. (1) Let $L \cong \mathbb{P}^{N-1} \subset \mathbb{P}^{N}$ be a general hyperplane. Since $X \subset \mathbb{P}^{N}$ is an aCM surface if and only if a general hyperplane section $C=X \cap L$ of $X$ is an aCM curve, it is enough to check that indeed $C \subset L=\mathbb{P}^{N-1}$ is an aCM curve. We observe that

$$
\operatorname{deg}(C)=\operatorname{deg}(X)=m^{2}+m+1 .
$$

By the adjunction formula we have:

$$
2 g(C)-2=C\left(C+K_{X}\right)=C^{2}-3
$$


(because $\left.C K_{X}=L K_{X}=\left((m+2) H-\sum_{i=1}^{3 m+3} E_{i}\right)\left(-3 H+\sum_{i=1}^{3 m+3} E_{i}\right)=-3\right)$. Therefore, $C^{2}=2 g(C)+1$. Thus, $\mathcal{O}_{C}(C)$ is a very ample line bundle on $C$ and by the Corollary to [19, Theorem 6], $\mathcal{O}_{C}(C)$ is projectively normal. So, $\mathcal{O}_{C}(C)$ embeds $C$ in a projective space as a projectively normal curve. Since $X$ is rational, it is regular (i.e. $\left.H^{1}\left(X, \mathcal{O}_{X}\right)=0\right)$ and from the exact cohomology sequence associated to the exact sequence

$$
0 \longrightarrow \mathcal{O}_{X} \longrightarrow \mathcal{O}_{X}(C) \longrightarrow \mathcal{O}_{C}(C) \longrightarrow 0
$$

we deduce that the projective space into which $\mathcal{O}_{C}(C)$ embeds $C$ is just $L$ and we are done.

(2) Let us first compute the dimension of $I(X)_{2}$. By [28, Corollary 7.2.3] there are $\frac{(m-2)(m-1) m(m+5)}{8}$ quadrics involving the monomials $x y z(x, y, z)^{m-1}$. A straightforward computation shows that there are $\left(\begin{array}{c}m \\ 2\end{array}\right)$ quadrics involving $G_{x}$ (resp., $G_{y}$ and $G_{z}$ ) and the monomials in $x y z(x, y, z)^{m-1}$. Therefore $\operatorname{dim} I(X)_{2}=3\left(\begin{array}{c}m+2 \\ 4\end{array}\right)$. To prove that $I(X)$ is generated by quadrics and cubics, we consider the $h$-vector of $X$. Recall that the $h$-vector of $X$ is a sequence $\left(h_{X}(i)\right)_{i \geq 0}$ of non-negative integers satisfying:

(i) For all $i \geq 0, h_{X}(i)=\Delta^{3} H(X, i)$ where $H(X, i)=\operatorname{dim}\left(\mathbb{C}\left[y_{0}, \ldots y_{N}\right] / I(X)\right)_{i}$.

(ii) $\operatorname{deg}(X)=\sum_{i \geq 0} h_{X}(i)$.

(iii) If $\sigma$ is the least integer such that $h_{X}(i)=0$, then $I(X)$ is generated by forms of degree $\leq \sigma$.

Since $X$ is non-degenerate and $\operatorname{dim} I(X)_{2}=3\left(\begin{array}{c}m+2 \\ 4\end{array}\right)$, we have $h_{X}(0)=1, h_{X}(1)=$ $h_{X}(2)=N-2$. From the equality

$$
\operatorname{deg}(X)=m^{2}+m+1=\sum_{i \geq 0} h_{X}(i)=2 N-3+\sum_{i \geq 3} h_{X}(i),
$$

we conclude that $h_{X}(i)=0$ for $i \geq 3, \sigma=3$ and $I(X)$ is generated by forms of degree $\leq 3$. Let us now prove that $I(X)$ is not generated by quadrics i.e. at least one cubic is required. Since $X$ is an aCM surface, we know that the graded Betti numbers of $X$ and of a general hyperplane section $C=X \cap \mathbb{P}^{N-1}$ are equal; in particular, the degrees of a minimal set of generators of $C$ and $X$ coincide. So, it will be enough to check that a minimal set of generators of $I(C)$ contains a cubic. In [13, Theorem 2], Green and Lazarsfeld proved that $I(C)$ fails to be generated by quadrics if and only if $C$ is hyperelliptic or $\mathcal{O}_{C}(C)$ embeds $C$ with a trisecant line, i.e. $H^{0}\left(C ; C-K_{C}\right) \neq 0$ where $K_{C}$ denotes the canonical divisor of $C$. We consider the exact sequence

$$
0 \longrightarrow \mathcal{O}_{X} \longrightarrow \mathcal{O}_{X}(C) \longrightarrow \mathcal{O}_{C}(C) \longrightarrow 0
$$

and its associated exact cohomology sequence:

$0 \longrightarrow H^{0}\left(X ;-C-K_{X}\right) \longrightarrow H^{0}\left(X ;-K_{X}\right) \longrightarrow H^{0}\left(C ; \mathcal{O}_{C}\left(-K_{X}\right)\right) \longrightarrow H^{1}\left(X ;-C-K_{X}\right) \cdots$

From the adjunction formula $K_{C} \cong \mathcal{O}_{C}\left(C+K_{X}\right)$, we deduce that $H^{0}\left(C ; \mathcal{O}_{C}\left(-K_{X}\right)\right) \cong$ $H^{0}\left(C ; \mathcal{O}_{C}\left(C-K_{C}\right)\right)$. On the other hand, $H^{0}\left(X ;-C-K_{X}\right) \cong H^{0}\left(X ;-L-K_{X}\right) \cong$ $H^{0}(X ;-(m-1) H)=0$ and $H^{1}\left(X ;-C-K_{X}\right) \cong H^{1}\left(X ;-L-K_{X}\right) \cong H^{1}(X ; L)=0$ (since $|M|$ is non-special). Therefore, we have

$$
H^{0}\left(C ; \mathcal{O}_{C}\left(C-K_{C}\right)\right) \cong H^{0}\left(X ;-K_{X}\right) \cong H^{0}\left(X ; 3 H-\sum_{i=1}^{3 m+3} E_{i}\right) \cong \mathbb{C}
$$


and we are done.

Let us prove that $C_{m}$ is part of a minimal system of generators of $I(X)$. To this end, we first describe $I(X)_{2}$. Recall that $X$ is the image of the rational map

$$
\begin{gathered}
\varphi: \mathbb{P}^{2} \rightarrow \mathbb{P}^{N} \\
(x: y: z) \mapsto\left(m_{0}: m_{1}: \cdots: m_{N-3}: G_{x}: G_{y}: G_{z}\right),
\end{gathered}
$$

where $m_{i}=x^{i_{0}} y^{i_{1}} z^{i_{2}}$ with $i_{0}+i_{1}+i_{2}=m+2$ and $i_{0}, i_{1}, i_{2} \geq 1$. Fix homogeneous coordinates $\omega_{0}, \ldots, \omega_{N}$ in $\mathbb{P}^{N} . I(X)_{2}$ is generated by

- All $\frac{(m-2)(m-1) m(m+5)}{8}$ quadrics $\omega_{i} \omega_{j}-\omega_{k} \omega_{t}, 0 \leq i, j, k, l \leq N-3$ satisfying $i_{u}+j_{u}=$ $k_{u}+t_{u}$ for $u=0,1,2$.

- All $\left(\begin{array}{c}m \\ 2\end{array}\right)$ quadrics $\omega_{i} H_{y}-(-1)^{m} \omega_{k} H_{z}-\omega_{s} \omega_{N-2}$ (Analogous quadrics with $\omega_{N-1}$ and $\left.\omega_{N}\right)$ where $\omega_{s}=x^{s_{0}} y^{s_{1}} z^{s_{2}}, \omega_{i}=x^{s_{0}-1} y^{s_{1}+1} z^{s_{2}}$ and $\omega_{k}=x^{s_{0}-1} y^{s_{1}} z^{s_{2}+1}$ with $s_{0}+$ $s_{1}+s_{2}=m+2,2 \leq s_{0}$ and $1 \leq s_{1}, s_{2}$.

Note that all quadrics in $I(X)_{2}$ vanish on the plane $\Pi: \omega_{0}=\ldots=\omega_{N-3}=0$ while $C_{m}$ does not vanish on $\Pi$. Therefore $C_{m}$ is not a linear combination of the quadrics in $I(X)_{2}$.

The existence of a trisecant line $\ell$ for $C$ is now clear by the Green-Lazarsfeld criterion mentioned above. If $C=X \cap \mathbb{P}^{N-1}$ then the trisecant line to $C$ is the line $\mathbb{P}^{N-1} \cap \Pi$.

It remains to see that there is only one cubic in a minimal system of generators of $I(C)$. This follows from the fact that $H^{0}\left(C ; \mathcal{O}_{C}\left(C-K_{C}\right)\right)=\mathbb{C}$ established in (13), which means that the span of the unique effective divisor in the bundle $\mathcal{O}_{C}\left(C-K_{C}\right)$ is the unique trisecant line. Indeed, assume that $C$ has a unique trisecant line $\ell$ and call $C_{1}$ the union of $C$ and $\ell$. Then $C_{1}$ is a canonical singular curve and its ideal is generated by quadrics (Petri's theorem) which are the quadrics in the ideal of $I(C)$ (In fact, $h^{0}\left(C ; \mathcal{O}_{C}(2)\right)=h^{0}\left(C_{1} ; \mathcal{O}_{C_{1}}(2)\right)$ by Riemann-Roch theorem for singular curves (see [9, Example 18.3.4]). Hence, to cut out $C$ from $C_{1}$, it suffices to consider one cubic in the ideal of $I(C)$ which is not a linear combination of quadrics.

Remark 5.8. (1) We note that the unexpected curve $C_{R, m}$ in [27, Theorem 4.6] can be written

$$
\begin{aligned}
C_{R, m} & =x y z\left[( m + 1 ) \left(a\left(b^{m}+(-1)^{m+1} c^{m}\right) x^{m-1}\right.\right. \\
& \left.+b\left(a^{m}+(-1)^{m+1} c^{m}\right) y^{m-1}+c\left(a^{m}+(-1)^{m+1} b^{m}\right) z^{m-1}\right) \\
& \left.+\sum_{i=1}^{m-2}(-1)^{i+1}\left(\begin{array}{c}
m+1 \\
i+1
\end{array}\right)\left(a^{m-i} b^{i+1} x^{i} y^{m-i-1}+a^{m-i} c^{i+1} x^{i} z^{m-i-1}+b^{m-i} c^{i+1} y^{i} z^{m-i-1}\right)\right] \\
& +a^{m+1} G_{x}+b^{m+1} G_{y}+c^{m+1} G_{z}
\end{aligned}
$$

and this form does not involve monomials that are a product of $(x y z)^{2}$ and a monomial of degree $m-4$. So, let $J$ be the ideal generated by all coordinates $\omega_{i}$ corresponding to the monomials in $(x y z)^{2} \mu_{m-4}$, we can consider the projection $\pi_{J}$ and consider the map $\bar{\varphi}_{m}=\pi_{J} \circ \varphi: \mathbb{P}^{2} \rightarrow \mathbb{P}^{3 m-1}$. In this way the ideal of the image $\bar{X}=\pi_{J}(X)$ of this map is the elimination ideal of $I(X)$ and so also $I(\bar{X})$ is generated by quadrics and a unique cubic which turns out to be the same as in Proposition 5.7. With a simple calculation we get that the number of quadrics decreases to $\frac{1}{2}\left(5 m^{2}-m-12\right)$.

Moreover, $\bar{X}$ is the result of subsequent projections from external points. So, the degree is preserved and $\operatorname{deg}(\bar{X})=\operatorname{deg}(X)=m^{2}+m+1$.

Now we recall that $\operatorname{deg}(\bar{X})=\sum_{i} h_{X}(i)$ and, as we know the Hilbert function in degree 1 
and 2 , we can compute $h_{X}(1)$ and $h_{X}(2)$ so we get that $\sum_{i \geq 3} h_{X}(i)=-(m-2)(m-3)<0$ for any $m \geq 4$. So we deduce that the $h$-vector is not positive and $\bar{X}$ is not aCM. Actually, computations with Macaulay2 supports the conjecture that the h-vector is

$$
h=\left(1,3(m-1), 2 m^{2}-7 m+9,-\frac{3}{2}(m-2)(m-3), \frac{1}{2}(m-2)(m-3)\right) .
$$

(2) In Proposition 5.7 we have seen that for all $m \geq 4$ the image of the map $\varphi: \mathbb{P}^{2} \longrightarrow \mathbb{P}^{N}$ where

$$
\varphi=\left(x y z \mu_{d}: G_{x}: G_{y}: G_{z}\right)
$$

is a smooth surface $X$ of degree $m^{2}+m+1$, its ideal $I(X)$ is generated by quadrics and only one cubic and it is aCM. Using Macaulay2 we have checked what happens with their companion surfaces $X^{\prime} \subset \mathbb{P}^{3 m-1}$. Recall that $X^{\prime}$ is the image of $\psi: \mathbb{P}^{2} \longrightarrow \mathbb{P}^{3 m-1}$ where

$$
\begin{gathered}
\psi=\left(a^{m+1}, b^{m+1}, c^{m+1}, a^{m-1} b^{2}, \ldots, a^{2} b^{m-1}, a^{m-1} c^{2}, \ldots, a^{2} c^{m-1}, b^{m-1} c^{2}, \ldots, b^{2} c^{m-1},\right. \\
a\left(b^{m}-(-c)^{m}, b\left(a^{m}-(-c)^{m}\right), c\left(b^{m}-(-a)^{m}\right) .\right.
\end{gathered}
$$

Our computation, for $m \leq 8$, strongly supports that the following hold: $\psi$ is a birational map onto its image, the ideal of $X^{\prime}$ is generated by $\frac{1}{2}\left(5 m^{2}-5 m-12\right)$ quadrics and a unique cubic (unless for $m=4$ where 10 independent cubics are necessary). Moreover, the companion has degree $(m+1)^{2}$ and with the same arguments as for $\bar{X}$ we conjecture that the $h$-vector is

$$
h:\left(1,3(m-1), 2 m^{2}-5 m+9,-\frac{3}{2}(m-2)(m-3), \frac{1}{2}(m-1)(m-6)\right) .
$$

Therefore, $X^{\prime}$ is not an aCM surface. Indeed, the projective dimension of the coordinate ring of $X^{\prime}$ is equal to $\operatorname{codim}\left(X^{\prime}\right)+1$.

Summarizing, for $m \leq 8$, we have that $X \subset \mathbb{P}^{N}$ is an aCM projection of the Veronese surface $V_{2, m+2}$ in $\mathbb{P}^{\left(\begin{array}{c}m+4 \\ 2\end{array}\right)-1}$ while its companion surface $X^{\prime}$ is a non-aCM projection of the Veronese surface $V_{2, m+2}$ in $\mathbb{P}^{3 m-1}$. This example makes more intriguing Gröbner's problem: To determine when a projection of a Veronese variety $V_{n, d} \subset \mathbb{P}^{\left(\begin{array}{c}n+d \\ d\end{array}\right)-1}$ is aCM and when it is not aCM (see [14]).

Remark 5.9. If we consider the ideal generated only by the quadrics in $I(\bar{X})$ the degree increases by 1 . Instead if we consider the ideal generated only by the quadrics in $I\left(X^{\prime}\right)$, the degree is still the $(m+1)^{2}$.

Finally, we remark explicitly, that the embedding of $\bar{X}$ is never too positive.

Corollary 5.10. The surface $X \subset \mathbb{P}^{N}$ does not satisfy the $N_{2}$ condition of Green.

Acknowledgement. Our work began during Oberwolfach workshop on Lefschetz Properties in Algebra, Geometry and Combinatorics held in September/October 2020. We thank the MFO for providing, during the COVID-19 crisis, excellent working conditions for collaboration between on site and remote participants of the workshop.

The first two authors have been partially supported by GNSAGA-INDAM. The third author has been partially supported by PID2020-113674GB-I00. The last two authors were partially supported by National Science Centre, Poland, Opus Grant 2019/35/B/ST1/00723. Our collaboration was partially supported by National Science Centre, Poland, Harmonia Grant 2018/30/M/ST1/00148.

We thank the referees for numerous remarks and insights which helped us to improve and clarify our results and their presentation. 


\section{References}

[1] T. Bauer, G. Malara, T. Szemberg, and J. Szpond. Quartic unexpected curves and surfaces. Manuscripta Math., 161(3-4):283-292, 2020.

[2] L. Chiantini and J. Migliore. Sets of points which project to complete intersections, and unexpected cones. Trans. Amer. Math. Soc., 374(4):2581-2607, 2021. With an appendix by A. Bernardi, L. Chiantini, G. Dedham, G. Favacchio, B. Harbourne, J.Migliore, T. Szemberg and J. Szpond.

[3] D. Cook II, B. Harbourne, J. Migliore, and U. Nagel. Line arrangements and configurations of points with an unexpected geometric property. Compos. Math., 154(10):21502194, 2018.

[4] E. Davis and A. Geramita. Birational morphisms to $\mathbb{P}^{2}$ : An ideal-theoretic perspective. Math. Ann., 279:435-448, 1988.

[5] R. Di Gennaro, G. Ilardi, and J. Vallès. Singular hypersurfaces characterizing the Lefschetz properties. J. Lond. Math. Soc. (2), 89(1):194-212, 2014.

[6] A. V. Doria, S. H. Hassanzadeh, and A. Simis. A characteristic-free criterion of birationality. Adv. Math., 230(1):390-413, 2012.

[7] M. Dumnicki, L. Farnik, B. Harbourne, G. Malara, J. Szpond, and H. Tutaj-Gasińska. A matrixwise approach to unexpected hypersurfaces. Linear Algebra Appl., 592:113133,2020 .

[8] M. Dumnicki, A. Küronya, C. Maclean, and T. Szemberg. Rationality of Seshadri constants and the Segre-Harbourne-Gimigliano-Hirschowitz conjecture. Adv. Math., 303:1162-1170, 2016.

[9] W. Fulton. Intersection theory, volume 2 of Ergebnisse der Mathematik und ihrer Grenzgebiete. 3. Folge. A Series of Modern Surveys in Mathematics /Results in Mathematics and Related Areas. 3rd Series. A Series of Modern Surveys in Mathematics]. Springer-Verlag, Berlin, second edition, 1998.

[10] A. Geramita and A. Gimigliano. Generators for the defining ideal of certain rational surfaces. Duke Math. J., 62:61-83, 1991.

[11] A. V. Geramita, B. Harbourne, and J. Migliore. Star configurations in $\mathbb{P}^{n}$. J. Algebra, 376:279-299, 2013.

[12] D. Grayson and M. Stillman. Macaulay2, a software system for research in algebraic geometry. http://www.math.uiuc.edu/Macaulay2/, 2020.

[13] M. Green and R. Lazarsfeld. Some results on the syzygies of finite sets and algebraic curves. Compositio Math., 67(3):301-314, 1988.

[14] W. Gröbner. Über Veronesesche Varietäten und deren Projektionen. Arch. Math. (Basel), 16:257-264, 1965.

[15] B. Harbourne. Anticanonical rational surfaces. Trans. Amer. Math. Soc., 349(3):11911208, 1997.

[16] B. Harbourne. Birational morphisms of rational surfaces. J. Algebra, 190(1):145-162, 1997. 
[17] B. Harbourne, J. Migliore, U. Nagel, and Z. Teitler. Unexpected hypersurfaces and where to find them. Michigan Math. J., 70(2):301-339, 2021.

[18] J. E. Humphreys. Introduction to Lie algebras and representation theory, volume 9 of Graduate Texts in Mathematics. Springer-Verlag, New York-Berlin, 1978. Second printing, revised.

[19] D. Mumford. Varieties defined by quadratic equations. In Questions on Algebraic Varieties (C.I.M.E., III Ciclo, Varenna, 1969), pages 29-100. Edizioni Cremonese, Rome, 1970.

[20] M. Nagata. On the 14-th problem of Hilbert. Amer. J. Math., 81:766-772, 1959.

[21] F. Russo and A. Simis. On birational maps and jacobian matrices. Compositio Mathematica, 126:335-358, 2001.

[22] H. Schenck. Resonance varieties via blowups of $\mathbb{P}^{2}$ and scrolls. Int. Math. Res. Not. IMRN, (20):4756-4778, 2011.

[23] K. Schwede, D. Smolkin, S. H. Hassanzadeh, and C. Bott. RationalMaps: rational maps between varieties. Version 0.3. A Macaulay2 package available at https:// github.com/Macaulay2/M2/tree/master/M2/Macaulay2/packages.

[24] G. Staglianò. Cremona: rational maps between projective varieties. Version 5.1. A Macaulay2 package available at https://github.com/Macaulay2/M2/tree/master/ M2/Macaulay2/packages.

[25] G. Staglianò. A Macaulay2 package for computations with rational maps. The Journal of Software for Algebra and Geometry, 8, 2018.

[26] J. Szpond. Unexpected curves and Togliatti-type surfaces. Math. Nachr., 293:158$168,2020$.

[27] J. Szpond. Fermat-type arrangements, In: Stamate D., Szemberg T. (eds) Combinatorial Structures in Algebra and Geometry. NSA 2018. Springer Proceedings in Mathematics \& Statistics, vol 331. p. 161 - 182, Springer, Cham, 2020.

[28] J. Weyman. Cohomology of vector bundles and syzygies, volume 149 of Cambridge Tracts in Mathematics. Cambridge University Press, Cambridge, 2003.

Roberta Di Gennaro,

Dipartimento di Matematica e Applicazioni "Renato Caccioppoli"

Università degli Studi di Napoli Federico II

Via Cintia, Complesso Universitario Monte Sant'Angelo

80126 Napoli, Italy.

E-mail address: digennar@unina.it

Giovanna Ilardi,

Dipartimento di Matematica e Applicazioni "Renato Caccioppoli"

Università degli Studi di Napoli Federico II

Via Cintia, Complesso Universitario Monte Sant'Angelo

80126 Napoli, Italy.

E-mail address: giovanna.ilardi@unina.it 
Rosa Maria Miró Roig,

Facultat de Matemàtiques i Informàtica, Universitat de Barcelona, Gran Via des les Corts Catalanes 585, 08007 Barcelona, Spain.

E-mail address: miro@ub.edu

Tomasz Szemberg,

Department of Mathematics, Pedagogical University of Cracow, Podchorążych 2, PL-30084 Kraków, Poland.

E-mail address: tomasz.szemberg@gmail.com

Justyna Szpond,

Institute of Mathematics, Polish Academy of Sciences, Śniadeckich 8, PL-00-656 Warszawa, Poland.

E-mail address: szpond@gmail.com 